\title{
Teoría de la jerarquía financiera ¿aplica en las grandes empresas mexicanas?
}

\author{
Theory of financial hierarchy, does it apply in large Mexican Companies? \\ Beatriz Sauza-Ávila ${ }^{a}$, Jair González-Ramírez ${ }^{b}$, Suly S-Pérez-Castañeda ${ }^{c}$,Claudia B. Lechuga- \\ Canto $^{d}$, Dorie Cruz-Ramírez ${ }^{e}$,Blanca E. Hernández-Bonilla ${ }^{f}$
}

\begin{abstract}
:
For any company, regardless of its size, it is necessary that it be financed in order to continue carrying out its operations, this implies that it can decide whether to use its own resources to do so, such as the reinvestment of profits or requires external financing sources using financial institutions. or very likely to use the support of its suppliers, in the last of the cases that every large company will resort to is the issuance of shares to be able to finance itself, with the application of the hierarchy theory or also called pecking order we can know how it is financing for their proper decision making and, in this way, keep their finances healthy.
\end{abstract}

Keywords:

Financing, debt, profitability

\section{Resumen:}

Para toda empresa no importando su tamaño es necesario que se financie para poder seguir realizando sus operaciones, esto implica que pueda decidir si utiliza sus propios recursos para hacerlo, como por ejemplo la reinversión de utilidades o requiere de fuentes de financiamiento externas haciendo uso de instituciones financieras o muy probablemente utilice el apoyo de sus proveedores, en último de los casos a que toda grande empresa recurrirá es a la emisión de acciones para poder financiarse, con la aplicación de la teoría de la jerarquía o también llamada pecking order podemos conocer cómo se está financiando para su adecuada toma de decisiones y de esta manera mantener sus finanzas sanas.

\section{Palabras Clave:}

Financiamiento, deuda, rentabilidad.

\section{Introducción}

La presente investigación tiene como objetivo analizar una grande empresa comercial para saber si cumple con lo establecido en la teoría de la jerarquía financiera en periodo de tres años. Como lo señala Garrido (2016), existen pocos estudios realizados en países desarrollados que muestren evidencias en la aplicación de esta teoría. Los pioneros en estudiar lo relacionado con estructura de capital fueron Modigliani y Miller (1958), ellos lo que buscaron era conocer cómo se financian las empresas para poder llevar a cabo sus funciones, de acuerdo con la teoría de la jerarquía financiera se presume que las empresas se financian primero utilizando sus propios recursos, posteriormente utilizando endeudamiento externo o a través de la emisión de acciones.

Autor de Correspondencia, Universidad Autónoma del Estado de Hidalgo, https://orcid.org/0000-0002-7919-6792, Email: beatriz_sauza@uaeh.edu.mx

b Universidad Autónoma del Estado de Hidalgo, Email: go368214@uaeh.edu.mx

c Universidad Autónoma del Estado de Hidalgo, https://orcid.org/0000-0002-3763-9233, Email: sulysp@uaeh.edu.mx

d Universidad Autónoma del Estado de Hidalgo,Email: claublc@uaeh.edu.mx

e Universidad Autónoma del Estado de Hidalgo, https://orcid.org/0000-0002-7853-7655, Email: doriec@ uaeh.edu.mx

f Universidad Autónoma del Estado de Hidalgo, Email: blancaestelab2005 @ hotmail.com 


\section{Financiamiento}

Cuando se habla de financiamiento es muy probable que se tenga una idea de que se está hablando, sin embargo; es necesario definirlo, el financiamiento es el proceso por el que se proporciona capital a una empresa o persona para utilizar en un proyecto o negocio, es decir, recursos como dinero y crédito para que pueda ejecutar sus planes. En el caso de las compañías, suelen ser préstamos bancarios o recursos aportados por sus inversionistas. (BBVA, 2021).

Se designa el término de financiamiento al conjunto de recursos monetarios y de crédito que se destinarán a una empresa, actividad, organización o individuo para que los mismos lleven a cabo una determinada actividad o concreten algo, como por ejemplo la apertura de un negocio. (Ucha, F. 2009).

El financiamiento o financiación es el proceso de viabilizar y mantener en marcha un proyecto, negocio o emprendimiento específico, mediante la asignación de recursos (dinero o crédito específico, mediante la asignación de recursos capitales (dinero o crédito) para el mismo. Dicho más fácilmente, financiar es asignar recursos capitales a una iniciativa determinada. El financiamiento es un elemento clave en el éxito de cualquier proyecto o empresa, ya que involucra los recursos que se necesitarán para ponerlo en marcha. Todo proyecto requiere, de una u otra manera, de cierto margen de financiación. (Raffino, 2020).

Un término adecuado para el préstamo de capital para una empresa es el financiamiento o financiación, esto ocurre cuando una empresa recurre a un préstamo por terceros para poder seguir en operación sus actividades o poner en marcha algún proyecto. También podemos decir que el fin anciamiento es don de la empresa adquiere una deuda y un crédito, por lo regular a largo plazo que una entidad requiere adquirirla para poder invertirla en algún proyecto que este tenga planeado.

Financiación no solo se refiere a deuda externa, sino también depende de la procedencia que este venga es decir podemos hablar de un financiamiento de fondos ajeno, el cual se refiere como deuda esto proviene de personas externas a la empresa, a bancos. Por otro lado, tenemos el financiamiento de fondos propios, esto se refiere a toda aportación de los socios de la misma empresa. El tipo de financiamiento puede ser a corto o largo plazo según la empresa, si es a corto plazo es menor a un año y si lo adquiere a largo plazo es mayor a un año.

Una vez que se ha definido el financiamiento, considerándose algo esencial para toda empra, se procederá a definir las variables utilizadas en la investigación las cuales son: deuda, rentabilidad, tamaño, tangibilidad, oportunidad de crecimiento.

\section{Deuda y apalancamiento.}

Las empresas al financiarse pueden contraer deuda algunos autores como Santos (1999), consideran que para una empresa endeudarse es un buen negocio financiero pero siempre habrá riesgos. Por ello es necesario identificar lo que se entiende por deuda.

La deuda es una obligación que tiene una persona física o jurídica para cumplir sus compromisos de pago, fruto del ejercicio de su actividad económica. (Burguillo, 2015).

La deuda es un cargo que tiene una persona física o jurídica para ejecutar sus responsabilidades de pago del ejercicio de la actividad económica. Es la cantidad de dinero que debemos de pagar a una persona o entidad, por alguna contraprestación recibida. Se contrae unaobligación financieraque hay que afrontar. (Gonzalez, 2018).

Deuda es la obligación que contrae quien pide algo de reintegrar lo pedido con acuerdo a unas condiciones pactadas previamente. El que ha pedido es el deudor, el que ha entregado o prestado es el acreedor; lo entregado puede ser cualquier tipo de bien, tangible o intangible. La deuda no es la cantidad prestada, ni la cantidad adeudada, esos son conceptos diferentes, aunque es común que deuda y cantidad adeudada se usen indistintamente para hablar de lo segundo. (Carreira, 2011).

Algunos autores hacen mención que la deuda es la cantidad a pagar de una entidad o persona que esta debe por el monto solicitado, podemos decir a más conciencia que la deuda de una persona física o moral es la obligación o compromiso que estas tienen para pagar el préstamo solicitado, una deuda está formada por la cantidad a pagar esto se refiere al capital, el plazo o vencimiento que se tien e para devolverlo y en otros casos el interés que se cobra por el préstamo. El interés ya dependerá de cada institución en donde se halla adquirido el préstamo.

Ahora bien para este estudio es necesario relacionar la deu da con el apalancamiento, este término será utilizado como variable independiente esta será medida a través de un ratio financiero como lo sugiere en Rajan y Zigales (1995), citado por Garrido (2016) la definición más amplia del endeudamiento es la relación de pasivo total entre active total. 


\section{Rentabilidad}

La rentabilidad hace referencia a los beneficios que se han obtenido 0 se pueden obtener de una inversión. (Arias, 2019).

La rentabilidad es la relación que existe entre la utilidad y la inversión necesaria para alcanzarla, ya que mide tanto la efectividad de la gerencia de una empresa, demostrada por las utilidades obtenidas de las ventas realizadas y utilización de inversiones. (Zamora, 2008).

La rentabilidad es cualquier acción económica en la que se movilizan una serie de medios, materiales, recursos humanos y recursos financieros con el objetivo de obtener una serie de resultados. Es decir, la rentabilidad es el rendimiento que producen una serie de capitales en un determinado periodo de tiempo. Es una forma de comparar los medios que se han utilizado para una determinada acción, y la renta que se ha generado fruto de esa acción. (Aguirre, 2019)

En términos de finanzas la rentabilidad es la capacidad que tienen las empresas o las entidades para reportar ya sea productividad, utilidades o beneficios económicos du rante su periodo o anualmente. La rentabilidad también es conocida como la capacidad qu e tiene una empre para obtener beneficios económicos. El cálculo de la rentabilidad se expresa en porcentajes.

\section{Tangibilidad}

Los activos tangibles de una empresa se definen como propiedades que se utilizan por un periodo de tiempo en las operaciones de la entidad y no se tiene la finalidad de venderlas (Ecured, 2012), de acuerdo con Escalona (2020), hace mención que los activos tangibles son lo que tienen una figura física y que pueden ser visto y tocado.

\section{Tamaño}

De acuerdo con Titman y Wessels (1988), las grandes empresas tienen menos probabilidad a la quiebra, significando que las grandes empresas deberían estar más apalancadas, por tener suficientes activos que pueden respaldar de la duda.

\section{Oportunidad de crecimiento}

Una estrategia de crecimiento es el conjunto de acciones y planes que diseña una empresa con el fin de aumentar su participación de mercado al lograr desarrollar una ventaja estable y única en su entorno competitivo.

Así, la estrategia de crecimiento espera que la empresa aumente su nivel de crecimiento al lograr aumentar su cuota de venta, su margen de utilidad, su participación de mercado o la expansión de la empresa. (Quiroa, 2019).

Por lo tanto, el crecimiento futuro esperado debería estar relacionado negativamente con los niveles de deuda a largo plazo (Titman, Wessels, 1988).

\section{Teoría de la jerarquía o también llamada peking order}

La teoría de la jerarquía se define como una de las teorías de mayor referencia dentro del apalancamiento de las empresas; manifiesta que la selección adversa incita a que las empresas prefieran una financiación interna antes que una externa (Myers, 1984) y a la vez declara que cuando se opta por la deuda externa se busca aquella que comprometa en un menor riesgo a la firma, principalmente en la calidad de la deuda y los costos de financiamiento. (Shyam Sunder y Myers, 210)

La teoría de la jerarquía basa sus supuestos afirmando que no existe una estructura óptima de capital sino que más bien los gerentes usan la jerarquía de preferencias a la hora de realizar nuevas inversiones, es decir primero se recurre al financiamiento con fondos internos debido a que en ésta no existe asimetría de la información, luego se recurre al endeudamiento y como última alternativa a la emisión de acciones (Corredor, 2013)

La teoría de financiamiento de la jerarquía de preferencias. Según los postulados de esta teoría, lo que determina la estructura financiera de las empresas es la intención de financiar nuevas inversiones, primero internamente con fondos propios, a continuación, con deuda de bajo riesgo de exposición como la bancaria, posteriormente con deu da pública en el caso que ofrezca menor subvaluación que las acciones y en último lugar con nuevas acciones. (Herrera Santiago, Limón Suárez y Soto lbáñez, 2006)

La teoría de la Jerarquía financiera la cual hace énfasis en algunos aspectos el cual dice que el encargado de la empresa o gerente empresarial tratan de incrementar sus inversiones, pero esta teoría en concreto se puede decir que es aquella donde la empresa buscara primero el financiarse por fuentes internas en este caso por ellas mismas, una vez agotada esta posibilidad la empresa buscara el financiarse por fuentes externas es decir a un endeudamiento de terceros y por ultimo a la emisión de acciones.

\section{Metodología}

El tipo de investigación es descriptivo al tomar como referencia los estados financieros de una empresa comercial, no experimental y transversal al considerar los tres años 2017,2018 y 2019, que la base de datos yahoo! Finanzas permite tener acceso a esta información financiera, el muestreo para esta investigación es por conveniencia, y por tener el acceso a los estado 
financieros en una plataforma libre es decir que no requiere de realizar alguna cuota para poder extraer los datos.

A continuación se presenta la Figura 1 del estado financiero llamado balance, correspondiente a la empresa comercial, el cual se utilizó para determinar las variables.

\begin{tabular}{|c|c|c|c|}
\hline \multicolumn{4}{|c|}{ Balance de la empresa comercial } \\
\hline & 2019 & 2018 & 2017 \\
\hline \multicolumn{4}{|l|}{ Activos } \\
\hline \multicolumn{4}{|l|}{ Activos corrientes } \\
\hline \multicolumn{4}{|l|}{ Efectivo } \\
\hline Caja y equivalentes de caja & $6,251,000$ & $7,584,000$ & $7,216,000$ \\
\hline Caja total & $6,251,000$ & $7,584,000$ & $7,216,000$ \\
\hline Cuentas pendientes netas & $17,128,000$ & $19,630,000$ & $18,565,000$ \\
\hline Inventario & $9,819,000$ & $9,340,000$ & $8,368,000$ \\
\hline Activos corrientes totales & $44,197,000$ & $44,851,000$ & $42,490,000$ \\
\hline \multicolumn{4}{|l|}{ Activos no corrientes } \\
\hline \multicolumn{4}{|l|}{ Propiedad, planta y equipo } \\
\hline Propiedad bruta, planta y equipo & $182,806,000$ & $153,723,000$ & $145,228,000$ \\
\hline Depreciación acumulada & $-72,915,000$ & $-66,480,000$ & $-62,256,000$ \\
\hline Propiedad neta, planta y equipo & $109,891,000$ & $87,243,000$ & $82,972,000$ \\
\hline Valor Ilave & $62,794,000$ & $65,513,000$ & $63,426,000$ \\
\hline Activos intangibles & $51,318,000$ & $54,476,000$ & $56,194,000$ \\
\hline Otros activos a largo plazo & $1,887,000$ & $1,281,000$ & $1,966,000$ \\
\hline Total de activos no corrientes & $234,884,000$ & $218,465,000$ & $216,759,000$ \\
\hline Activos totales & $279,081,000$ & $263,316,000$ & $259,249,000$ \\
\hline \multicolumn{4}{|l|}{ Patrimonio neto y accionistas } \\
\hline \multicolumn{4}{|l|}{ Obligaciones } \\
\hline \multicolumn{4}{|l|}{ Pasivos circulantes } \\
\hline Deuda corriente & $5,408,000$ & $1,153,000$ & $2,766,000$ \\
\hline Cuentas a pagar & $24,169,000$ & $21,983,000$ & $19,751,000$ \\
\hline Otros pasivos circulantes & & & $2,307,000$ \\
\hline Pasivos circulantes totales & $54,620,000$ & $48,749,000$ & $48,655,000$ \\
\hline \multicolumn{4}{|l|}{ Obligaciones no corrientes } \\
\hline Deuda a largo plazo & $81,264,000$ & $88,693,000$ & $91,546,000$ \\
\hline Obligaciones de impuestos diferidas & $5,241,000$ & $5,720,000$ & $4,682,000$ \\
\hline Otras obligaciones a largo plazo & $3,661,000$ & $9,347,000$ & $6,704,000$ \\
\hline Total de obligaciones no corrientes & $146,150,000$ & $129,992,000$ & $133,570,000$ \\
\hline Pasivos totales & $200,770,000$ & $178,741,000$ & $182,225,000$ \\
\hline \multicolumn{4}{|l|}{ Patrimonio neto } \\
\hline Acción ordinaria & $4,156,000$ & $4,199,000$ & $4,227,000$ \\
\hline Ganancias retenidas & $61,332,000$ & $59,238,000$ & $60,180,000$ \\
\hline Total de patrimonio neto de los accionistas & $73,736,000$ & $79,690,000$ & $72,767,000$ \\
\hline Total de obligaciones y capital de los accionistas & $279,081,000$ & $263,316,000$ & $259,249,000$ \\
\hline
\end{tabular}

Figura 1. Estado financiero

Fuente: Yahoo! Finanzas 2021

\section{Variables proxy}

Las variables que se utiliza son de acuerdo a lo que se aprecia en la tabla 1., servirán para determinar la deuda, la tangibilidad, rentabilidad, tamaño y oportunidad de crecimiento, con ello se puede obtener una aproximación si la empresa en estudio cumple con lo establecido con la teoría de la jerarquía.

\begin{tabular}{|l|l|}
\hline Variable dependiente & \\
\hline Deuda $=$ & Total pasivos / total activos \\
\hline Variables independientes \\
\hline Tangibles = & $\begin{array}{l}\text { Activo fijo neto / Total } \\
\text { activos }\end{array}$ \\
\hline Rentabilidad = & EBIT / Total activos \\
\hline Tamaño $=$ & Log (total activos) \\
\hline $\begin{array}{l}\text { Oportunidad de } \\
\text { Crecimiento }=\end{array}$ & $\begin{array}{l}\text { Valor de mercado del } \\
\text { patrimonio / Patrimonio }\end{array}$ \\
\hline
\end{tabular}

Tabla 1 Variables

Fuente: Garrido (2016)

\section{Resultados}

De acuerdo con Myers y Majluf establecieron un modelo en el cual explican los comportamientos del financiamiento corporativo, tomando en consideración las fuentes internas de fondos, posteriormente preferir la deuda al capital social si se requiere financiamiento externo, después de este gran aprendizaje de estos autores pioneros en analizar la teoría de la jerarquía financiera, surgen otros au tores los cuales establecen que al medir con variables proxy utilizando ratios financieros los cuales para este estudio se obtuvieron los resultados de la rentabilidad, el tamaño, la tangibilidad, oportunidad de crecimiento y el apalancamiento arrojando los resultados presentados en la tabla 2.

Tabla 2. Matriz de variables proxy

\begin{tabular}{|c|r|r|rrr|}
\hline AÑN & RENTABILDAD & TAMAÑO & TANGIBIIDAD & $\begin{array}{c}\text { OPORTUNIDAD } \\
\text { DECRECIMIENTO }\end{array}$ & APALANCAMIENTO \\
\hline 2017 & 0.0901 & 19.4470126 & 0.841633791 & 2.20943908 & 0.719396878 \\
\hline 2018 & 0.0956 & 19.3888654 & 0.829668535 & 2.065513866 & 0.678807972 \\
\hline 2019 & 0.0829 & 19.3732995 & 0.836103514 & 2.277109129 & 0.702895672 \\
\hline
\end{tabular}

Fuente: Elaboración propia de los estados financiero de una empresa comercial

Una vez que se determinan los ratios financieros de la tabla 2 se procedió a realizar la correlación que existe entre el apalancamiento como variable independiente y la rentabilidad, tamaño, tangibilidad, oportunidad de crecimiento y apalancamiento como variables dependientes, obteniéndose los resultados mostrados en la tabla 3. 


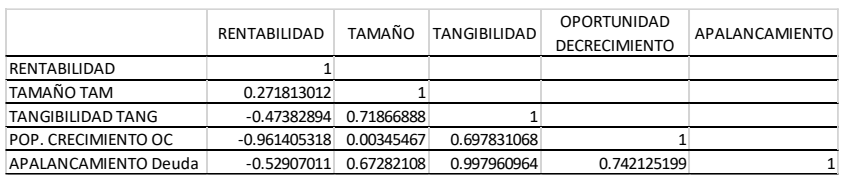

Tabla 3. Matriz de correlación

Fuente: Elaboración propia de los estados de una empresa comercial

La relación que se presenta entre apalancamiento y rentabilidad se comporta como lo establece la teoría de la jerarquía financiera según Garrido (2016), el tamaño y el apalancamiento muestra una relación positiva la empresa analizada tiende a endeudarse más una justificación como lo dice Titman y Wessels (1988), porque cuentan con suficientes activos fijos para poder ponerlos como garantía, con relación a la tangibilidad y el apalancamiento también muestra una relación positiva, se observa también una relación positiva entre la oportunidad de crecimiento y el apalancamiento.

\section{Conclusiones}

Como hace mención Garrido (2016), se han aplicado los estudios de la teoría de la jerarquía a países desarrollados y existen pocos estudios en países en vías de desarrollo, por ello al realizar esta investigación con la finalidad de conocer el financiamiento de las grandes empresas mexicanas que sirva para la adecuada toma decisiones evitando poner en riesgo a la organización.

Gracias a este estudio se pudo aplicar la teoría de la jerarquía y obtener que en la empresa comercial, si se aplica la teoría de la jerarquía financiera al obtener como resultado en la relación negativa entre el apalancamiento y rentabilidad, aún falta incorporar más estudios en estos países que permita alcanzar lo estándares de los países desarrollados.

\section{Referencias}

Aguirre, J. (2019). MytripleA. Obtenido de https://www.mytriplea.com/diccionario-financiero/rentabilidad/

Arias, A. S. (2019). Economipedia. Obtenido de https://economipedia.com/definiciones/rentabilidad.html

BBVA. (2021). BBVA Information. Obtenido de https://www.bbva.mx/educacion-

financiera/f/financiamiento.html

Burguillo, R. V. (2015). Economipedia. Obtenido de https://economipedia.com/definiciones/deuda.html

Carreira, F. (06 de Julio de 2011). El blog salmon. Obtenido de https://www.elblogsalmon.com/conceptos-de-economia/quees-deuda
Corredor, S. M. (2013). Teoría del Pecking Order versus teoría del Trade off para la empresa Coservicios S.A. E.S.P. Apuntes del Cenes.

Ecured (2012), Activo fijo tangible, consultada el 10 de febrero de 2021 en https://www.ecured.cu/Activo_fijo_tangible.

Escalona A. (2020). Activos tangibles: ¿qué son? ¿cuáles son? Y más, consultado el 10 de febrero de 2021 en https://tumundoeconomia.com/contabilidad/activos-tangibles/ Gonzalez, $\underset{\text { https://www.billin.net/glosario/definicion-deuda/ }}{\text { P. }}$ (2018).

Myers S.C, Majluf N.S, Corporate financing and investment decisión when firms have information that investors do not have. The Journal of Financial Economics, 1984; 13 (0): 187-221.

Quiroa, M. (2019). Economipedia. Obtenido de https://economipedia.com/definiciones/estrategia-decrecimiento.html

Raffino, M. E. (03 de Septiembre de 2020). Concepto de. Obtenido de https://concepto.de/financiamiento/

Samuel Mongrut, D. F. (2010). Explorando teorías de estructura de capital en Latinoamérica. Valoración de empresas en Latinoamérica, 173-184.

Santos N, (1999), ¿es negocio endeudarse? Industria Data UNMSN, facultad de ingeniería industrial, 2 (1) 2-5.

Shyam, Sunder, y Myers. (2010). Pecking Order behavior in emergin markets. Journal of International Financial Management \& Accounting, 21, 1, 31 .

Titman S, Wessels R, The Determinats of Capital Structure Choice. The Journal of finance, 1988;43 (1): 1-19.

Ucha, F. (23 de marzo de 2021) https:// www. definicionabc.com/ economia/financiamiento.php

Yahoo! Finanzas. (2021). Financieros, consultada el 8 de enero de 2021,

Zamora, A.I. (2008). Rentabilidad y ventaja comparativa: un análisis de los sistemas de producción de guayaba en el Estado de Michoacán. Universidad Michoacana de San Nicolas Hidalgo. 